\title{
Revisiting benzene cluster cations for the chemical ionization of dimethyl sulfide and select volatile organic compounds
}

\author{
Michelle J. Kim ${ }^{1, a}$, Matthew C. Zoerb ${ }^{2, b}$, Nicole R. Campbell ${ }^{2}$, Kathryn J. Zimmermann ${ }^{2, c}$, Byron W. Blomquist ${ }^{3}$, \\ Barry J. Huebert ${ }^{4}$, and Timothy H. Bertram ${ }^{2, d}$ \\ ${ }^{1}$ Scripps Institution of Oceanography, University of California San Diego, La Jolla, CA, USA \\ ${ }^{2}$ Department of Chemistry and Biochemistry, University of California San Diego, La Jolla, CA, USA \\ ${ }^{3}$ University of Colorado, Cooperative Institute for Research in Environmental Sciences, Boulder, CO, USA \\ ${ }^{4}$ School of Oceanography and Earth Sciences and Technology, University of Hawaii, Manoa, Honolulu, HI, USA \\ ${ }^{a}$ now at: Division of Geological and Planetary Sciences, California Institute of Technology, Pasadena, CA, USA \\ b now at: Department of Chemistry and Biochemistry, California Polytechnic \\ State University, San Luis Obispo, CA, USA \\ ${ }^{c}$ now at: School of Science and Technology, Georgia Gwinnett College, Lawrenceville, GA, USA \\ ${ }^{d}$ now at: Department of Chemistry, University of Wisconsin-Madison, Madison, WI, USA
}

Correspondence to: Timothy H. Bertram (tbertram@chem.wisc.edu)

Received: 20 July 2015 - Published in Atmos. Meas. Tech. Discuss.: 1 October 2015

Revised: 26 February 2016 - Accepted: 2 March 2016 - Published: 5 April 2016

\begin{abstract}
Benzene cluster cations were revisited as a sensitive and selective reagent ion for the chemical ionization of dimethyl sulfide (DMS) and a select group of volatile organic compounds (VOCs). Laboratory characterization was performed using both a new set of compounds (i.e., DMS, $\beta$-caryophyllene) as well as previously studied VOCs (i.e., isoprene, $\alpha$-pinene). Using a field deployable chemical-ionization time-of-flight mass spectrometer (CIToFMS), benzene cluster cations demonstrated high sensitivity (> $1 \mathrm{ncps} \mathrm{ppt}^{-1}$ ) to DMS, isoprene, and $\alpha$-pinene standards. Parallel measurements conducted using a chemicalionization quadrupole mass spectrometer, with a much weaker electric field, demonstrated that ion-molecule reactions likely proceed through a combination of ligandswitching and direct charge transfer mechanisms. Laboratory tests suggest that benzene cluster cations may be suitable for the selective ionization of sesquiterpenes, where minimal fragmentation $(<25 \%)$ was observed for the detection of $\beta$ caryophyllene, a bicyclic sesquiterpene. The in-field stability of benzene cluster cations using CI-ToFMS was examined in the marine boundary layer during the High Wind Gas Exchange Study (HiWinGS). The use of benzene cluster cation chemistry for the selective detection of DMS was validated against an atmospheric pressure ionization mass spectrom-
\end{abstract}

eter, where measurements from the two instruments were highly correlated $\left(R^{2}>0.95,10 \mathrm{~s}\right.$ averages $)$ over a wide range of sampling conditions.

\section{Introduction}

Volatile organic compounds (VOCs) play a central role in atmospheric chemistry by regulating tropospheric ozone and secondary organic aerosol (SOA) production rates (Goldstein and Galbally, 2007). Global emissions of non-methane VOCs are dominated by biogenic VOCs (BVOCs) such as terpenes, where isoprene $\left(\mathrm{C}_{5} \mathrm{H}_{8}\right)$ and monoterpenes $\left(\mathrm{MT} ; \mathrm{C}_{10} \mathrm{H}_{16}\right)$ emissions have been shown to be most significant (Arnold et al., 2009). Recent studies have shown that sesquiterpenes (SQT; $\mathrm{C}_{15} \mathrm{H}_{24}$ ) may also play a significant role in secondary organic aerosol production (Jaoui et al., 2013 and others), but far less is known about their global emission rates (Guenther et al., 2012; Kanakidou et al., 2005). In one of the few studies with simultaneous measurements of isoprene, MT, and SQT, Kim et al. (2009) inferred ecosystem scale fluxes that suggest that SQT fluxes could be as much as $50 \%$ that of MT in deciduous forests (Kim et al., 2009). In terrestrial regions, the condensable oxidation products of terpenes 
has been shown to drive SOA production, particularly in boreal and subtropical forests (Guenther et al., 2012). In comparison, SOA precursors in the marine boundary layer have historically been thought to be dominated by dimethyl sulfide (DMS) emissions, with isoprene and monoterpenes contributing less than $1 \%$ to SOA mass (Myriokefalitakis et al., 2010). The following paper focuses on the development of a chemical-ionization procedure for targeting marine trace gas emissions (e.g., DMS, isoprene, and monoterpenes) that, following oxidation, may have consequent impacts on aerosol particle mass loadings and size distributions.

Chemical-ionization mass spectrometry (CIMS) is increasingly utilized as a fast, sensitive, and selective measurement technique for in situ detection of reactive trace gas species (Huey, 2007). The benefits of CIMS are most pronounced when sampling from moving platforms or during instrumentally tasking sampling methods such as eddy covariance, where high time resolution is required. Proton transfer mass spectrometry (PTR-MS) has been used extensively for the sensitive, selective determination of VOCs providing direct measurements of concentrations and turbulent fluxes from a variety of mobile platforms (e.g., Lindinger et al., 1998). PTR-MS is well suited for the measurement of small molecules such as DMS and isoprene, but fragmentation of larger volatile organic compounds, such as monoterpenes and sesquiterpenes, can limit quantitative measurement (Kim et al., 2009). In parallel, separation techniques (e.g., gas chromatography) have been utilized to detect BVOCs with good sensitivity with the added benefit of resolving isobaric interferences at both nominal and fragment masses (Helmig et al., 2004). However, low temporal resolution limits their utility for higher time resolution analyses. In what follows, we extend the early work of Leibrock and Huey (2000) to explore the utility of benzene cluster cations, $\left(\mathrm{C}_{6} \mathrm{H}_{6}\right)_{n}^{+}$, for the selective, sensitive, and rapid detection of various SOA precursors via CIMS in the marine boundary layer.

The use of benzene cations as selective reagent ions dates back to at least the work of Horning et al. (1973), where trace benzene vapor was introduced into an atmospheric pressure ionization mass spectrometer. As discussed by Horning, benzene ion chemistry was thought to proceed through either a charge transfer reaction (R1) if the ionization energy (IE) for the analyte (A) was less than that of benzene $(9.24 \mathrm{eV})$ or through a proton transfer reaction (R2) if the gas-phase basicity of the analyte (B) is greater than that of the phenyl radical:

$$
\begin{aligned}
& \left(\mathrm{C}_{6} \mathrm{H}_{6}\right)^{+}+\mathrm{A} \rightarrow\left(\mathrm{C}_{6} \mathrm{H}_{6}\right)+\mathrm{A}^{+} \\
& \left(\mathrm{C}_{6} \mathrm{H}_{6}\right)^{+}+\mathrm{B} \rightarrow\left(\mathrm{C}_{6} \mathrm{H}_{5}\right)+\mathrm{BH}^{+}
\end{aligned}
$$

Horning et al. (1973) demonstrated that large, complex organics (e.g., testosterone) could be detected with minimal fragmentation as the molecular ion $\left(\mathrm{M}^{+}\right)$, while 2,6dimethyl- $\gamma$-pyrone was detected as $\mathrm{MH}^{+}$following $\mathrm{R} 2$. It is thus expected that an array of gas-phase bases (e.g., amines) could be detected using this ion chemistry. With specific application to atmospheric chemistry, Ketkar et al. (1991) revisited benzene ion chemistry for the selective detection of 2-chloroethyl ethyl sulfide.

The most complete and recent work on benzene ion chemistry, with application to atmospheric measurements, is from two papers by Leibrock and Huey, where the potential for benzene cations in the detection of isoprene and monoterpenes, among a suite of other conjugated dienes and aromatics, was discussed. Leibrock and Huey (2000) demonstrated that select VOCs with ionization energies lower than that for benzene $(9.24 \mathrm{eV})$ react at or near the collision limit either via direct charge transfer (R1) forming the charge transfer product $\mathrm{X}^{+}$or through a ligand switching reaction involving the benzene dimer cation (R3) forming the ion-neutral product $\mathrm{X}^{+}-\left(\mathrm{C}_{6} \mathrm{H}_{6}\right)$. It is likely that if ionization proceeds through R3, the number of molecules that can be detected will be reduced as the ionization energy for the benzene dimer cation has been measured to be significantly less (ca. $8.6 \mathrm{eV}$ ) than the monomer (Grover et al., 1987).

$\left(\mathrm{C}_{6} \mathrm{H}_{6}\right)_{2}^{+}+\mathrm{X} \rightarrow \mathrm{X}^{+}\left(\mathrm{C}_{6} \mathrm{H}_{6}\right)+\left(\mathrm{C}_{6} \mathrm{H}_{6}\right)$

It follows that benzene ion chemistry should be a sensitive measurement for a host of molecules of interest to the study of marine air, including DMS $(\mathrm{IE}=8.6 \mathrm{eV})$, monoterpenes $(\mathrm{IE}=8.07 \mathrm{eV}, \alpha$-pinene), sesquiterpenes $(\mathrm{IE}=8.3 \mathrm{eV}$, $\beta$-caryophyllene), and their first-generation oxidation products, as well as a host of atmospheric amines (through R2) (Al-Joboury and Turner, 1964; Hunter and Lias, 1998; McDiarmid, 1974). The high density of states of $\mathrm{C}_{6} \mathrm{H}_{6}$ and $\left(\mathrm{C}_{6} \mathrm{H}_{6}\right)_{2}$ may also permit the efficient formation of weakly bound ion-neutral clusters, as collisional energy from the ion-molecule reactions (IMRs) can be readily absorbed. However, the extent to which this is important is governed by the energetics of the specific IMR.

These advantages are not without potential challenges. These challenges include the extensive dehydration of alcohols can complicate the interpretation of mass spectra. Leibrock and Huey showed that the dehydration of 2-methyl3-buten-2-ol (MBO) could lead to a positive artifact in the detection of isoprene, despite the fact that the IE for MBO exceeds $9.24 \mathrm{eV}$. In addition, benzene ion chemistry is not exceedingly selective as compared with other ion-molecule chemistries, resulting in the potential for interferences in low mass resolution instruments, particularly in polluted air masses. Finally, while it is not expected that benzene cations will directly ionize water, it has been shown that under high specific humidity, benzene cations may have a series of attached water molecules (Ibrahim et al., 2005; Miyazaki et al., 2004) that may introduce a water dependence in the ion chemistry.

In what follows, we describe laboratory characterization experiments and field observations in the remote marine boundary layer to assess the utility of benzene cations for 
the detection of DMS and select BVOCs. Laboratory experiments were conducted using both a chemical-ionization time-of-flight mass spectrometer (CI-ToFMS) (Bertram et al., 2011) and a chemical-ionization quadrupole mass spectrometer (CI-QMS) to probe IMR mechanisms and adduct stability under different electric field strengths. Field measurements from the remote North Atlantic boundary layer were validated against simultaneous measurements of DMS made by an atmospheric pressure ionization mass spectrometer with an isotopically labeled standard (APIMS-ILS) (Blomquist et al., 2010).

\section{Experimental}

\subsection{Instrument descriptions}

The utility of benzene reagent-ion chemistry for the sensitive, selective detection of DMS, isoprene, and monoterpenes was characterized on two CIMS instruments: (1) a field deployable CI-ToFMS (Tofwerk AG and Aerodyne Research Inc.) (Bertram et al., 2011) optimized for the sensitive detection of trace gases from mobile platforms and (2) a laboratory-based CI-QMS operated without declustering optics or ion guides, achieving low field electric strengths at the cost of sensitivity. Despite different ion transmission optics and mass analyzers, the two instruments utilize identical IMR chambers.

\subsubsection{Chemical-ionization time-of-flight mass spectrometer}

A complete description of the CI-ToFMS hardware can be found in Bertram et al. (2011). Briefly, reagent ions are generated by passing benzene gas diluted in ultra-high purity (UHP) $\mathrm{N}_{2}$ through an $\alpha$-emitting polonium source (Sect. 2.1.3), then mixed with ambient air in an IMR chamber $\left(P_{\mathrm{IMR}}=70 \mathrm{mbar}\right)$. Reaction times are estimated to be on the order of $100 \mathrm{~ms}$, significantly longer than the timescale for most weakly bound clusters (e.g., water or benzene) to reach equilibrium. Product ions pass through four additional differentially pumped stages, beginning with the collisional dissociation chamber $\left(P_{\mathrm{CDC}}=2 \mathrm{mbar}\right)$ housing an RF-only quadrupole ion guide. In the CDC, ion-molecule adducts can be dissociated by adjusting the electric field strength or pressure of the chamber. Ions are then passed through the second RF-only quadrupole ion guide housed at $1.4 \times 10^{-2}$ mbar, and then a chamber containing final focusing optics that condition the ion beam for entry into the extraction region of the ToF mass analyzer, where ions are orthogonally extracted into a compact time-of-flight mass spectrometer (Tofwerk AG and Aerodyne Research Inc.). Typical extraction frequencies range between 30 and $85 \mathrm{kHz}$ resulting in mass spectra spanning between 11 and $319 \mathrm{~m} / Q$. Individual mass spectra are averaged and saved at $10 \mathrm{~Hz}$ following the procedures outlined in Bertram et al. (2011).

\subsubsection{Chemical-ionization quadrupole mass spectrometer}

The CI-QMS used in these studies employs the same ion molecule reaction chamber described in Bertram et al. (2011), but operated at a different pressure $\left(P_{\mathrm{IMR}}=100 \mathrm{mbar}\right)$. In contrast to the CI-ToFMS, the CIQMS was operated to minimize the declustering of weakly bound ion-neutral clusters prior to detection. The CI-QMS was operated with only three stages of differential pumping and with a series of lenses in place of the RF ion guide devices. The resulting instrument achieves minimal collisional declustering at the expense of sensitivity. Ions were mass selected using a quadrupole mass analyzer (Extrel Inc.). Transmitted ions were detected using a continuous dynode electron multiplier, where single ion events were converted to TTL pulses for measurement and analysis via custom LabView software. The CI-QMS was operated in parallel with the CI-ToFMS to provide insight into the ion cluster distributions at the point of reaction as the stronger electric fields, and subsequent dissociation of ion-neutral clusters, in the CIToFMS limit our ability to monitor ion chemistry occurring in the IMR.

\subsubsection{Reagent-ion generation and chemistry}

On both platforms, the reagent-ion precursor (benzene gas) was generated by diluting a certified compressed gas standard $\left(150 \pm 0.1 \mathrm{ppm}\right.$ in $\mathrm{N}_{2}$, Praxair) with UHP $\mathrm{N}_{2}$ generated from LN2 boil off. The total reagent flow rate is limited by an inline critical orifice $\left(1.8 \mathrm{sL} \mathrm{min}^{-1}\right)$ which also serves the additional purpose of keeping permeable Teflon tubing at positive pressure relative to the atmosphere to prevent diffusion of contaminants into the ion generation lines. Downstream of the orifice, gas flow is passed through an $\alpha$ emitting polonium-210 source (NRD 2021-1000 at $10 \mathrm{mCi}$ initial activity). $\mathrm{N}_{2}^{+}$ions are formed via electron transfer reactions with $\alpha$ particles (R4) and go on to ionize neutral benzene (Grover et al., 1987) to form a distribution of benzene cations $\left(\mathrm{C}_{6} \mathrm{H}_{6}\right)_{n}^{+}(n=1,2, \ldots)(\mathrm{R} 5-6)$.

\section{Laboratory characterization}

\subsection{Factors controlling primary ion distributions}

During laboratory experiments, the CI-QMS and CI-ToFMS ion generation systems were identical with the exception of the IMR pressure. As shown in Fig. 1, the benzene ion distribution as measured by CI-QMS was detected predominantly in the form of $\left(\mathrm{C}_{6} \mathrm{H}_{6}\right)_{2}^{+}$at $156 \mathrm{~m} / Q$ (Fig. 1a). The same gas mixture was detected by CI-ToFMS in the form of $\mathrm{C}_{6} \mathrm{H}_{6}^{+}$at $78 \mathrm{~m} / \mathrm{Q}$ (Fig. 1b). This suggests that the reagent-ion distribution primarily exists as $n \geq 2$ in the CI-ToFMS IMR as well, but the field strength of the RF-only quadrupole ion guide in the CDC of the CI-ToFMS exceeds the benzene dimer bind- 
ing energy of $15.3 \mathrm{kcal} \mathrm{mol}^{-1}$ (Grover et al., 1987) despite operating at a relatively weak field strength. Unfortunately, even in the CI-QMS, significant declustering occurs between the IMR and the mass analyzer. As a result, it is not possible to comment directly on the distribution of benzene cluster cations in the IMR and the possibility that the clusters may have attached water molecules. However, based on the CIQMS spectra, it is most likely that the clusters are at least dimers $(n \geq 2)$ in both instruments.

\subsubsection{Dependence on benzene reagent gas concentration}

Background mass spectra were examined by overflowing the sample inlet with UHP zero air while varying the concentration of benzene delivered through the ionizers of both the CIToFMS and the CI-QMS instruments. The relative humidity at the sample inlet was less than $5 \%$ during these experiments. On the CI-ToFMS, the benzene monomer accounted for $68-91 \%$ of the total ion current (Fig. 2) for benzene precursor ion concentrations ranging between 1 and $45 \mathrm{ppm}$. Peak benzene ion signal intensities were found at benzene reagent gas concentrations of approximately $10 \mathrm{ppm}$. At lower benzene concentration $(<10 \mathrm{ppm})$, a wide array of background ions are prevalent in the mass spectra. Although the zero air cylinders were assumed to be nominally dry, trace amounts of water (detected as protonated water clusters) were evident in the sample stream at low benzene concentrations. At higher benzene concentrations (>10 ppm) background peaks at 92,106 , and $120 \mathrm{~m} / Q$ become an increasing fraction of the total ion current. At $10 \mathrm{ppm}$ benzene, these ions collectively account for 1-3\% of total ion current. During High Wind Gas Exchange Study (HiWinGS) underway measurements, the sum of these three peaks comprised an average of $2.6 \%(1 \sigma=0.8 \%)$ of the total ion current. These background peaks were observed across all CIMS instruments and their strength increased with benzene concentration (Fig. 2), suggesting they are trace contaminants in the benzene cylinders. We provisionally attribute these peaks to toluene, ethylbenzene, xylene, and trimethylbenzene.

As shown in Fig. 3, the ratio of benzene cation dimer to monomer was approximately $1: 10^{4}$ on the CI-ToFMS and $3 \times 10^{3}: 1$ for the CI-QMS. The presence of the dimer in the CI-ToFMS mass spectrum provides additional evidence that the reagent-ion distribution primarily exists as $n \geq 2$ in both of the IMRs, but the field strength of the RF-only quadrupole ion guide in the CDC of the CI-ToFMS exceeds the benzene dimer binding energy of $15 \mathrm{kcal} \mathrm{mol}^{-1}$ (Grover et al., 1987).

\subsubsection{Dependence on ambient humidity}

Ambient humidity was varied to probe its impact on ion chemistry and product ion distributions. In this experiment, benzene reagent gas concentrations were held at $10 \mathrm{ppm}$ while sampling humid UHP zero air $(0-100 \%$ relative hu-

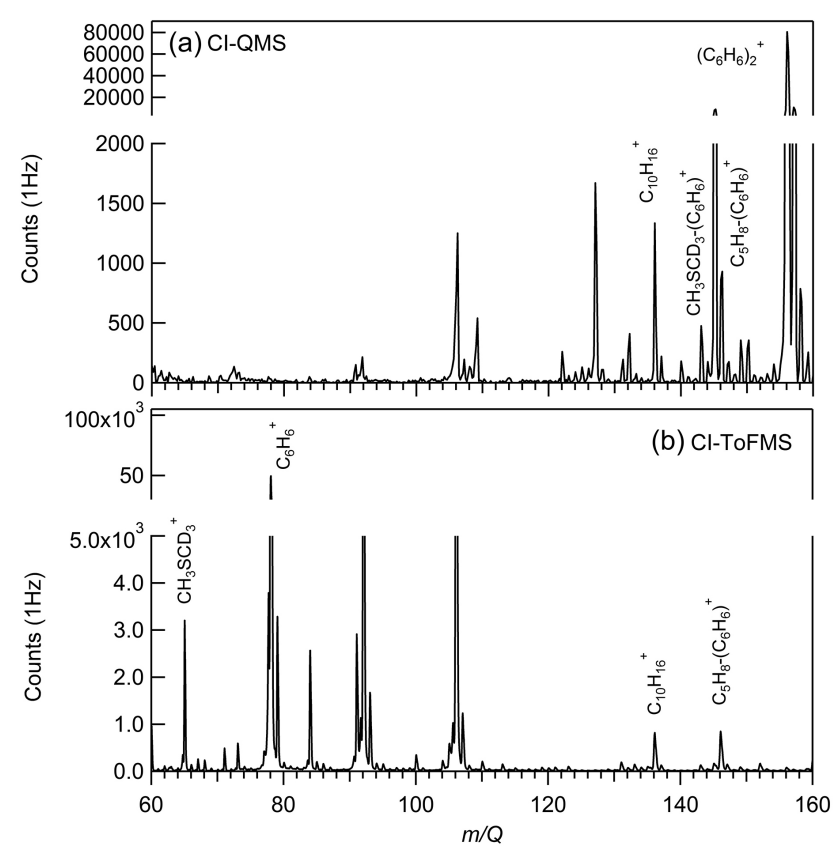

Figure 1. CI-QMS (a) and CI-ToFMS (b) mass spectra utilizing benzene reagent-ion chemistry while sampling a mixture of dimethyl-1,1,1- $\mathrm{d}_{3}$ sulfide, isoprene, and $\alpha$-pinene standards at $40 \%$ relative humidity and room temperature. The weaker field strength of the CI-QMS transmits clustered forms of benzene $\left(\left(\mathrm{C}_{6} \mathrm{H}_{6}\right)_{2}^{+}\right)$ and dimethyl-1,1,1- $\mathrm{d}_{3}$ sulfide $\left(\mathrm{CH}_{3} \mathrm{SCD}_{3}-\mathrm{C}_{6} \mathrm{H}_{6}^{+}\right)$. Both are detected at nominal mass by the CI-ToFMS. Isoprene is detected as a benzene cluster $\left(\mathrm{C}_{5} \mathrm{H}_{8}-\mathrm{C}_{6} \mathrm{H}_{6}^{+}\right)$and $\alpha$-pinene $\left(\mathrm{C}_{10} \mathrm{H}_{16}^{+}\right)$appears at nominal mass on both instruments.

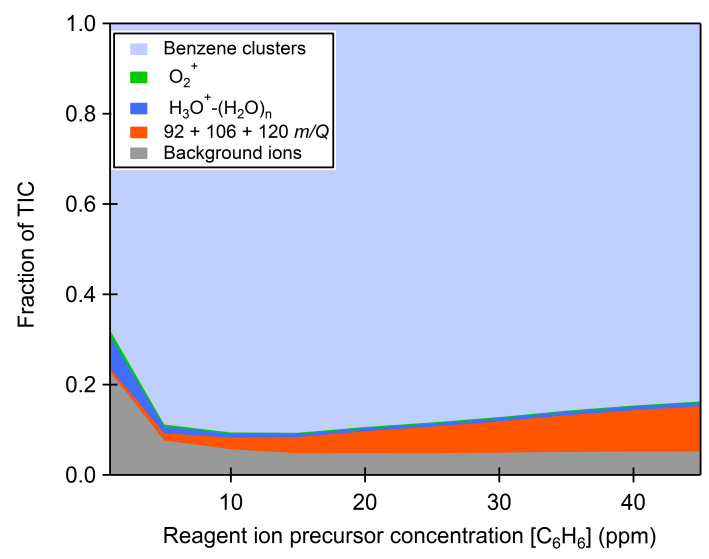

Figure 2. Partitioning of total ion current (TIC) on the CI-ToFMS between benzene clusters (light blue), $\mathrm{O}_{2}^{+}$(green), water clusters (dark blue, $n=0$ to 3 ), and background peaks (orange and gray) as a function of benzene reagent-ion concentration at $<1 \%$ relative humidity. Molecules corresponding to 92,106 , and $120 \mathrm{~m} / Q$ are attributed to alkyl-substituted benzene molecules: $\mathrm{C}_{6} \mathrm{H}_{5}-\mathrm{CH}_{3}^{+}$, $\mathrm{C}_{6} \mathrm{H}_{4}-\left(\mathrm{CH}_{3}\right)_{2}^{+}$, and $\mathrm{C}_{6} \mathrm{H}_{4}-\left(\mathrm{CH}_{3}\right)_{3}^{+}$, respectively.

midity, room temperature). On the CI-ToFMS, benzene monomers remained $>86 \%$ of the total ion current (Fig. $4 a$ ) 


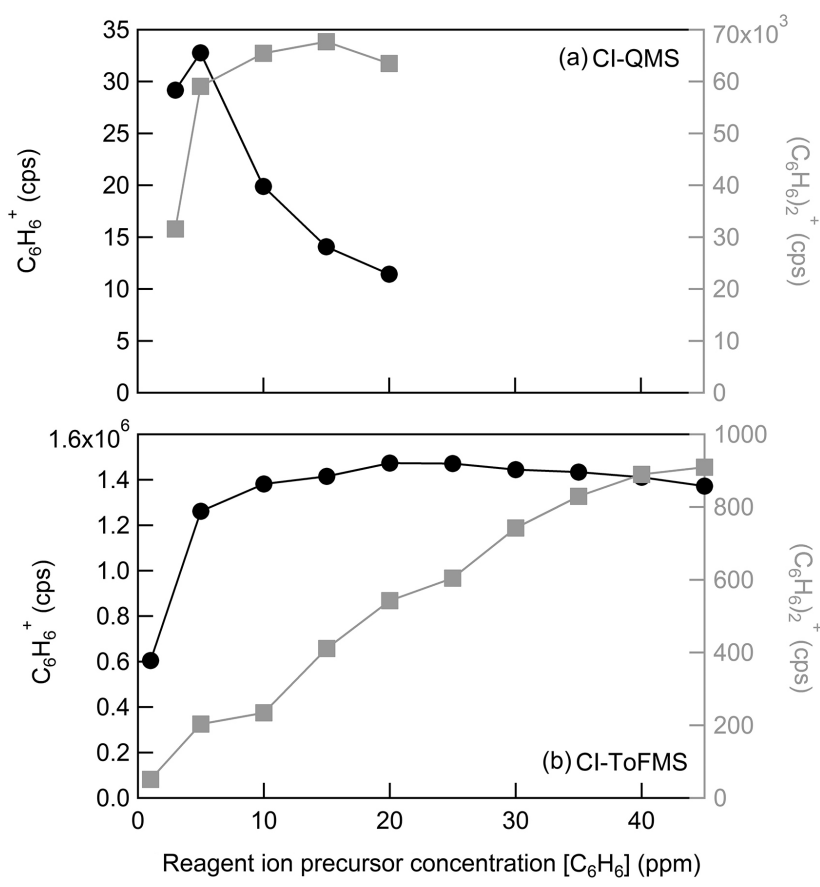

Figure 3. Dependence of benzene ion signal intensity on the benzene reagent gas concentration for both the CI-QMS (a) and CIToFMS (b). Nearly all CI-QMS benzene signal intensity is found at the dimer $(156 \mathrm{~m} / \mathrm{Q})$ while the CI-ToFMS was nearly exclusively in the form of the monomer $(78 \mathrm{~m} / \mathrm{Q})$. On both platforms, the dominant benzene peak plateau after $10 \mathrm{ppm}$ neutral benzene concentration.

at $100 \%$ relative humidity $(\mathrm{RH})$, compared with $91 \%$ at $<1 \% \mathrm{RH}$. Absolute benzene monomer count rates showed minimal sensitivity to ambient water vapor (Fig. 4b). Protonated water clusters $\left(\mathrm{H}_{3} \mathrm{O}^{+}-\left(\mathrm{H}_{2} \mathrm{O}\right)_{n}\right)$ were observed upon the addition of water vapor to sample air. The sum of the ion current for $\mathrm{H}_{3} \mathrm{O}^{+}-\left(\mathrm{H}_{2} \mathrm{O}\right)_{n}(n=0-3)$ ranged from 0.7 to $8.5 \%$ of the total ion current, increasing with relative humidity. Again, trace amounts of water vapor were detected while sampling nominally dry UHP zero air (Praxair) due to a small amount of condensation in zero air cylinders.

Given that the ionization energy of benzene $(9.2 \mathrm{eV}$ monomer, $8.69 \mathrm{eV}$ dimer) (Nemeth et al., 1993) is less than water (12.6 eV) (Page et al., 1988), it is unlikely that a benzene mediated pathway for the formation of protonated water clusters is active. It is possible that protonated water clusters may be formed by direct ionization (Good et al., 1970) due to back diffusion of ambient water into the Po-210 source, or persistent $\mathrm{N}_{2}^{+}$from the ionizer may contribute to direct ionization of water downstream in the IMR. Similarly, molecular oxygen ions $\left(\mathrm{O}_{2}^{+}, 12.0 \mathrm{eV}\right)$ (Tonkyn et al., 1991) from ambient air are observed in trace amounts $(<1.3 \%$ of the total ion current). However, despite the fact that benzene-water clusters were not observed in the mass spectra, we have no

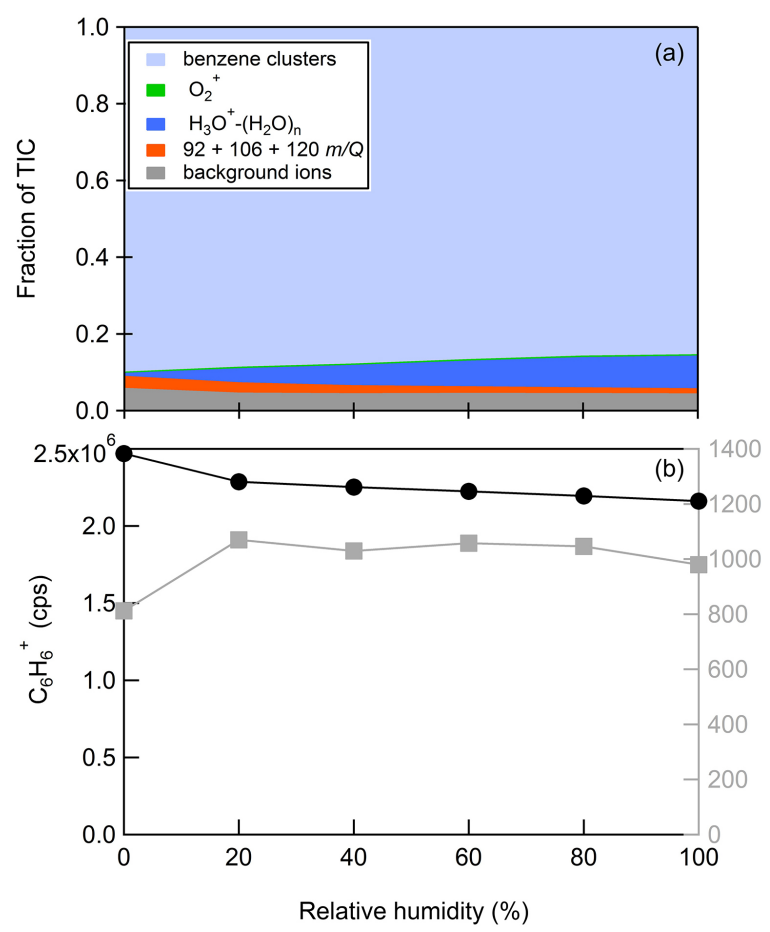

Figure 4. (Top) Dependence of primary ion signal intensities and partitioning between the monomer and dimer on ambient relative humidity $\left(\left[\mathrm{C}_{6} \mathrm{H}_{6}\right]=10 \mathrm{ppm}\right)$ on the CI-ToFMS. (Bottom) Absolute benzene ion signal intensities are shown to be nearly independent of ambient relative humidity.

direct evidence that they are not present in the IMR and they may impact instrument sensitivity, as described in Sect. 3.4.

\subsection{Instrument calibration}

Calibration curves were generated using three parallel CIMS systems (CI-ToFMS, CI-QMS, and CIMS that employs a residual gas analyzer for ion selection and quantification, CI-RGA) to determine the sensitivity of benzene cations to dimethyl sulfide, isoprene, and $\alpha$-pinene. Results from the first two instruments are included here. Results from the CIRGA will be discussed in a forthcoming manuscript. Calibration factors were determined using atmospherically relevant mixing ratios (0 to $3.2 \mathrm{ppb}$ ) of dimethyl-1,1,1- $\mathrm{d}_{3}$ sulfide (Praxair certified standard, $0.184 \times 10 \%$ ), isoprene (Praxair certified standard, $0.500 \times 10 \%$ ), and $\alpha$-pinene (Praxair certified standard, $0.497 \times 10 \%)$ at specific humidities $(q)$ from 0 to $16.6 \mathrm{~g} \mathrm{~kg}^{-1}$. The primary VOC calibration standards were diluted with UHP zero air (Praxair). Humidified buffer gas was generated by flowing UHP zero air through three sequential frit bubblers (Ace Glass) containing deionized water. Flow rates for all VOC standards and both the dry and saturated dilution flows were measured using mass flow meters or mass flow controllers $( \pm 10 \%)$. A mass closure calculation was performed during portions of the experiments 


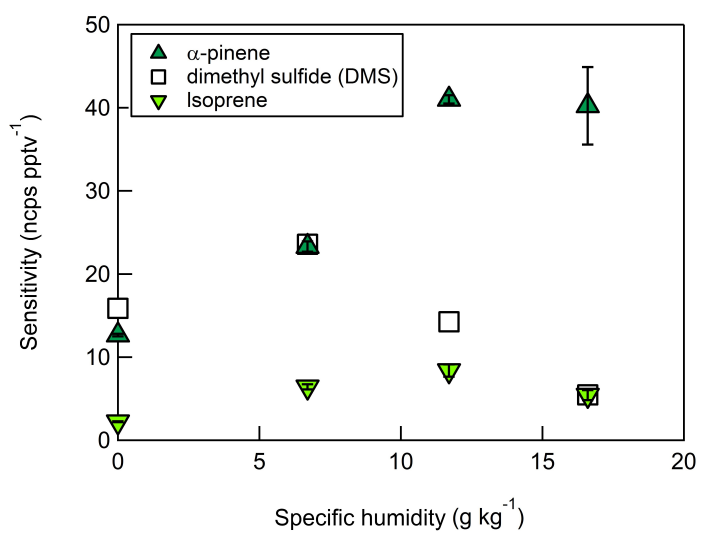

Figure 5. Humidity dependence of the laboratory-derived CIToFMS sensitivities to dimethyl-1,1,1- $\mathrm{d}_{3}$ sulfide, $\alpha$-pinene, and isoprene. Error bars indicate $1 \sigma$ as determined from the slope of the calibration curve.

using measured flow rates and saturation vapor pressures to ensure the humidified air stream was fully saturated. Bubbler temperatures were recorded for specific humidity calculations accounting for cooling at very high flow rates due to gas expansion. All CIMS instruments were positioned around a central manifold $(0.635 \mathrm{~cm}$ OD PFA tube and $0.635 \mathrm{~cm}$ PFA Swagelok fittings) and sampled from its own radial branch. Humidified air was added furthest downstream to minimize the surface area exposed to condensation. Each inlet branch was the same length to within $5 \mathrm{~cm}$. Excess flow was directed from the central manifold line to an exhaust at atmospheric pressure.

Benzene cations were demonstrated to be sensitive to isoprene and $\alpha$-pinene (Fig. 5) in agreement with previous work by Leibrock and Huey (2000), as well as DMS. Normalized sensitivities (normalized counts per second per ppt or ncps $\mathrm{ppt}^{-1}$ ) were calculated by adjusting product ion count rates to a reference reagent-ion signal of $10^{6} \mathrm{cps}$ (Warneke et al., 2001). Thus, absolute sensitivity increases with benzene ion current. Calibration factors are reported as the slope of the linear fit of each calibration curve $(1 \sigma=$ slope standard deviation). Maximum normalized sensitivities were as follows: DMS (23.5 ncps ppt $\left.{ }^{-1}\right), \alpha$-pinene $\left(41 \mathrm{ncps} \mathrm{ppt}^{-1}\right)$, and isoprene $\left(8.3 \mathrm{ncps} \mathrm{ppt}^{-1}\right)$. All three VOCs calibration factors exhibited unique humidity dependences described in more detail in Sect. 3.3.

\subsection{Adduct declustering}

VOC product ions were detected at nominal mass $\left(\mathrm{M}^{+}\right)$or as a benzene adduct $\left(\mathrm{M}-\left(\mathrm{C}_{6} \mathrm{H}_{6}\right)^{+}\right)$. On both the CI-QMS (Fig. 1a) and CI-ToFMS (Fig. 1b) isoprene was detected as an adduct $(146 \mathrm{~m} / Q)$, while $\alpha$-pinene appeared at nominal mass $(136 \mathrm{~m} / Q)$. The former indicates that isoprene ionization proceeds through a ligand switching reaction. For the latter, this result suggests that $\alpha$-pinene may also undergo a ligand switching mechanism with subsequent disassociation during transmission or, alternatively, may be ionized via direct charge transfer.

In contrast, dimethyl-1,1,1- $\mathrm{d}_{3}$ sulfide was detected predominately as $\mathrm{M}^{+}(65 \mathrm{~m} / \mathrm{Q})$ on the CI-ToFMS and as a benzene-DMS adduct $(143 \mathrm{~m} / Q)$ on the CI-QMS. This indicates that DMS ionization proceeds through a ligandswitching reaction in both instruments, but the adduct dissociates in the stronger declustering fields of the CI-ToFMS. Despite attempts to minimize the field strength of the CIToFMS, we were not able to observe the DMS-benzene cluster.

Instrument response to pure, unquantified mixing ratios of a host of other VOCs, including a sesquiterpene and a monoterpenoid, were assessed with the CI-ToFMS. In these experiments a small volume $(<1 \mathrm{~mL})$ of pure $\beta$ caryophyllene and eucalyptol standards were deposited into clean flasks and their vapors were delivered to the CI-ToFMS inlet. Product ions were identified and normalized (\%) to the sum of all feasible products. As described previously, DMS and $\alpha$-pinene product ions appear at $\mathrm{M}^{+}$(99 and $83 \%$, respectively), while isoprene is detected primarily as a benzene adduct ( $88 \%$ ) (Fig. 6). $\beta$-caryophyllene was detected as $\mathrm{M}^{+}(204 \mathrm{~m} / Q)$ with very limited fragmentation $(<25 \%)$. Since $\beta$-caryophyllene has been demonstrated to fragment more readily that other sesquiterpenes (Kim et al., 2009), these tests suggest that benzene dimer cations show excellent promise as an accurate chemical-ionization method to target easily fragmented terpene species. Eucalyptol (1,3,3-Trimethyl-2-oxabicyclo[2,2,2]octane), an oxygenated monoterpenoid $\left(\mathrm{C}_{10} \mathrm{H}_{18} \mathrm{O}\right)$, was also detected as $\mathrm{M}^{+}$ $(154 \mathrm{~m} / Q)$ with limited fragmentation (Fig. 6). In the case of eucalyptol, a secondary ionization reaction was evident at the $\mathrm{M}-\mathrm{H}^{+}$channel (Maleknia et al., 2007). After accounting for contributions from the carbon-13 isotope, we estimate the ratio of charge transfer to proton transfer product ions is about $2: 1$ at ambient RH for eucalyptol.

\subsection{Impact of ambient humidity on sensitivity}

The sensitivity of benzene cluster cations to DMS, $\alpha$-pinene, and isoprene standards was shown to be a strong function of specific humidity $(q)$ (Fig. 5), with peak sensitivity occurring at specific humidities of $6.7 \mathrm{~g} \mathrm{~kg}^{-1}$ (DMS, $23 \mathrm{ncps} \mathrm{ppt}^{-1}$ ), $11.7 \mathrm{~g} \mathrm{~kg}^{-1}$ ( $\alpha$-pinene, $41 \mathrm{ncps} \mathrm{ppt}^{-1}$ ), and $11.7 \mathrm{~g} \mathrm{~kg}^{-1}$ (isoprene, $8.3 \mathrm{ncps} \mathrm{ppt}^{-1}$ ). Sensitivity varied with ambient specific humidity on all CIMS platforms. On the CI-ToFMS, DMS was observed to be least sensitive to changes in ambient water vapor, while $\alpha$-pinene sensitivity increased almost linearly for $q$ values between 0 and $11.7 \mathrm{~g} \mathrm{~kg}^{-1}$.

At present, we do not have a definitive interpretation of the water dependence. While we do not observe a benzenewater cluster in any of our instruments, it is likely that the cluster exists in the IMR and is broken apart in the ion optics between the IMR and the mass analyzer. Results from 


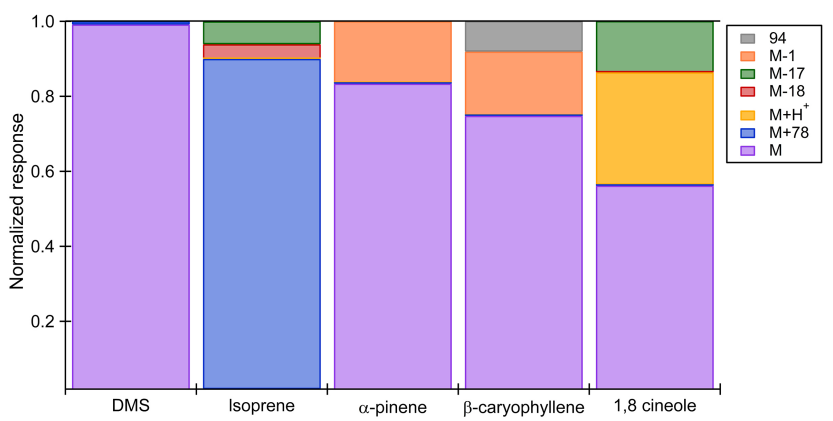

Figure 6. Normalized response of the CI-ToFMS to a series of VOC standards. Significant products (>5\% of instrument response) are shown for DMS, isoprene, $\alpha$-pinene, $\beta$-caryophyllene, and eucalyptol (1,8-cineole). For eucalyptol, the ratio of charge transfer to proton transfer products was roughly $2: 1$. Expected contributions from carbon-13 isotopes were accounted for in the calculated proton transfer products.

Ibrahim et al. (2005) show that the stepwise binding energies for attaching a water molecule to $\mathrm{C}_{6} \mathrm{H}_{6}^{+}$are on the order of $8.5 \mathrm{kcal} \mathrm{mol}^{-1}$, suggesting that the water molecules are removed via collisions in the transfer optics. Laboratory studies have shown previously that benzene-water clusters $\left[\mathrm{C}_{6} \mathrm{H}_{6}-\right.$ $\left.\left(\mathrm{H}_{2} \mathrm{O}\right)_{n}\right]^{+}$can be made efficiently $(n=1-23)$ (Miyazaki et al., 2004). For large clusters $(n \geq 4)$ IR spectra of the clusters suggest features that are nearly identical to protonated water clusters, suggesting exclusion of benzene and the formation of an attached water cluster. For smaller clusters $(n \leq 3)$, IR spectra were shown to be consistent with a hydrated benzene cation, where benzene retains over $90 \%$ of the charge (Ibrahim et al., 2005). It is likely that the sensitivity dependence we observe in these experiments is related to the aforementioned laboratory studies and depends on the location of the charge (benzene vs. water) and the size of the benzenewater cluster $(n)$, both of which are related to $q$. However, in our experiments it is almost certain that the reagent ion contains more than one $\mathrm{C}_{6} \mathrm{H}_{6}$ molecule limiting the direct comparison between the two studies. In what follows, we show that standard additions under a range of atmospheric values for $q$ are required for accurate measurements of mixing ratios in the atmosphere.

\section{CI-ToFMS performance during the High Wind Gas Exchange Study}

The CI-ToFMS utilizing benzene cluster cation ion chemistry was deployed to the Arctic and North Atlantic aboard the R/V Knorr during the fall of 2013 to characterize BVOCs in the remote marine boundary layer. Here, we analyze instrument performance over the 5-week HiWinGS to investigate factors controlling reagent-ion stability and sensitivity to VOCs in challenging environmental conditions. The atmospheric implications of these measurements will be discussed in a separate forthcoming manuscript. Isoprene, $\alpha$-pinene, and UHP zero air gas cylinders were used to determine infield sensitivities and system baselines, respectively. We validate our DMS measurement against a collocated DMS measurement made by the University of Hawaii's APIMS-ILS.

\subsection{Inlet characterization}

A low-pressure (200 mbar), rapid-response, high Reynolds number (ca. 2400) inlet assembly was designed based on the inlet manifold described in Ellis et al. (2010) and adapted for measurement of VOC mixing ratios and air-sea exchange from research vessels. The complete inlet assembly included separate lines for sample, bypass, baseline, and calibration flows sheathed together by a durable weatherproof exterior ( $25 \mathrm{~m}$ total length, $18 \mathrm{~m}$ weatherproofed, $6 \mathrm{~cm}$ outside diameter; Clayborn Labs). All inlet lines were connected to a glass manifold (Ellis et al., 2010). Inlet flow rates are set by a critical orifice (ca. $1.2 \mathrm{~mm} \mathrm{ID,} 12.56 \mathrm{sL} \mathrm{min}^{-1}$ ) set within the $2.54 \mathrm{~cm}$ OD cylindrical glass body. Upstream manifold surfaces were treated with Fluoropel (Cytonix). Downstream of the orifice (low-pressure side), sample flow is split into a bypass (ca. $2.5 \mathrm{sL} \mathrm{min}^{-1}$ ) and sample (ca. $10 \mathrm{sL} \mathrm{min}^{-1}$ ) stream. The bypass branch is in-line with the manifold intake. The sample flow follows a series of 90 and $180^{\circ}$ turns, which serve to eliminate super-micrometer sea spray particles from the sample inlet stream. The manifold was housed in an insulated, weather-proofed housing and mounted off the foremast. The sample intake was ca. $20 \mathrm{~m}$ above mean sea level (a.m.s.l.) and ca. $17 \mathrm{~m}$ aft of the bow (Yang et al., 2014). The entire inlet sample line and manifold assembly was heated by a single PID heating circuit (Omega) set to $40^{\circ} \mathrm{C}$.

The inlet assembly comprised of a sample $(0.95 \mathrm{~cm}$ OD FEP), bypass $0.635 \mathrm{~cm}$ OD PFA), baseline $(0.95 \mathrm{~cm}$ OD FEP), and calibration ( $0.318 \mathrm{~cm}$ OD FEP) line and ran between the manifold and a temperature-controlled instrument van housing the CI-ToFMS on the 02 deck. Baseline and calibration gas flows were controlled by mass flow controllers operated by an automated LabView program. Ambient sample air was pulled through the sample line to the front block, an additional differentially pumped chamber upstream of the CI-ToFMS IMR and held at the inlet assembly pressure $(200 \mathrm{mbar})$. The CI-ToFMS subsampled from the front block, while the rest of the flow rejoined the bypass flow downstream of the instrument. Choked flow conditions in the entire manifold-inlet-front block volume were maintained by a $500 \mathrm{~L} \mathrm{~min}^{-1}$ scroll pump (Varian TriScroll 600). Inlet assembly pressures were recorded by a Pirani pressure gauge on the front block. UHP zero air and VOC standards were added to the inlet manifold via separate ports located upstream of the critical orifice (i.e., high-pressure side) to account fully for all losses to the manifold and inlet surfaces. Instrument baselines were determined by overflowing the manifold critical orifice with $20 \mathrm{sL} \mathrm{min}^{-1}$ of UHP zero air from a gas tank (Praxair). VOC sensitivity was assessed with several daily standard additions and calibrations. The first 3 


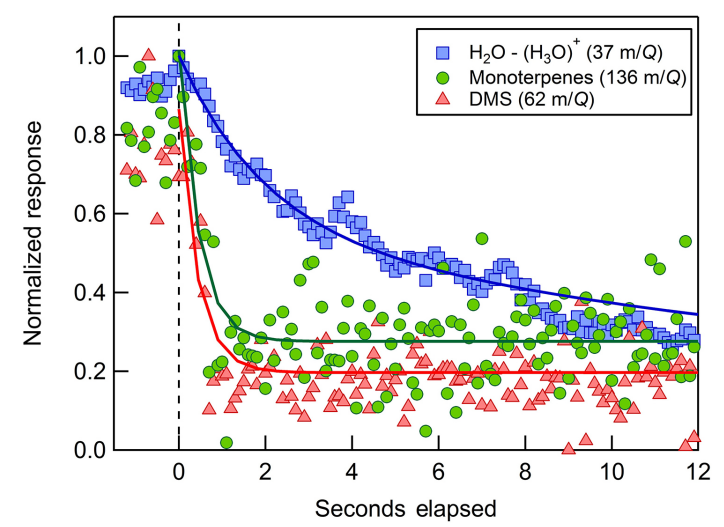

Figure 7. Inlet and instrument response to a prompt switch from ambient sampling to a zero air addition. The first rapid decay is attributed to the gas evacuation time $\left(\tau_{1}\right)$ and the second decay is attributed to the inlet wall-equilibration time $\left(\tau_{2}\right)$. Best fit estimates of $\tau_{1}$ for $\mathrm{H}_{3} \mathrm{O}^{+}\left(\mathrm{H}_{2} \mathrm{O}\right)$, DMS, and monoterpenes ranged between 2 and $3 \mathrm{~s}$, in agreement with the volumetric evacuation time of the sample inlet (3.6 seconds). $\tau_{2}$ was determined to be $22,1.2$, and $2.5 \mathrm{~s}$, respectively.

weeks of the research cruise utilized isoprene standards before switching to $\alpha$-pinene during the last 2 weeks. For both calibrations, a $0.500 \mathrm{ppm}$ primary standard in $\mathrm{N}_{2}$ (Praxair) was used to achieve a constant $638 \mathrm{ppt}$ mixing ratio in the inlet.

\subsection{Inlet time response}

Ambient VOC signal decays resulting from zero air additions were examined to characterize the inlet's time response (Fig. 7). Fitting a double exponential curve to signal decays yields two characteristic time constants for the inlet (Ellis et al., 2010). The first exponential decay time, $\tau_{1}$, is attributed the gas evacuation time and related to the physical operation and characteristics of the inlet itself. The second exponential decay time, $\tau_{2}$, is attributed to the equilibration constant of the gas and its polarity and solubility. For the $n=1$ protonated water cluster $\left(\mathrm{H}_{3} \mathrm{O}^{+}-\left(\mathrm{H}_{2} \mathrm{O}\right)_{n}\right), \tau_{1}$ of $3.2 \mathrm{~s}$ agreed well with the volumetric evacuation time of $3.6 \mathrm{~s}$ at $12.56 \mathrm{sL} \mathrm{min}^{-1}$. The second characteristic time, $\tau_{2}$ was long for the protonated water cluster at $22 \mathrm{~s}$, as expected based on the polarity and high surface affinity of water vapor. DMS and $\alpha$-pinene also exhibited fast $\tau_{1}$ time constants, but $\tau_{2}$ time constants were also fast (1.2 and $2.5 \mathrm{~s}$, respectively), in contrast to $\mathrm{H}_{2} \mathrm{O}$, indicating turbulent flow and minimal interaction of the analyte with the inlet walls.

\subsection{Factors impacting reagent-ion stability}

Benzene reagent-ion signal varied between 58 and $89 \%$ of the total ion current $\left(8.02 \times 10^{5}\right.$ to $6.4 \times 10^{6} \mathrm{cps}$ benzene $)$ during HiWinGS. Probability density functions of benzene counts during ambient and baseline periods each showed

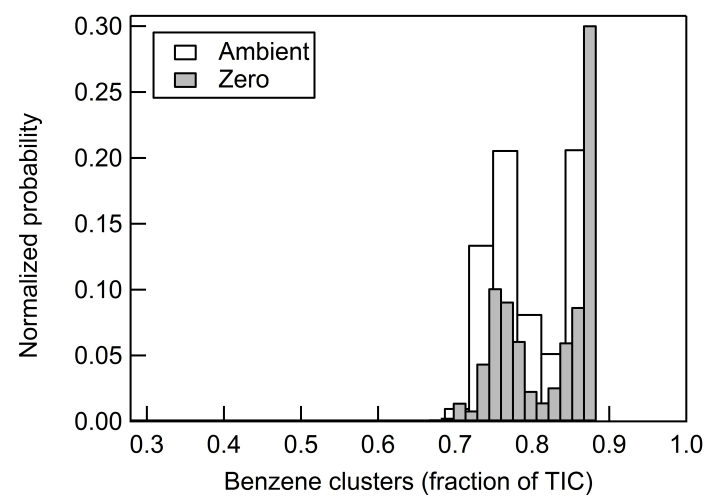

Figure 8. Probability density functions of benzene signals (\% total ion current, $1 \mathrm{~Hz}$ ) for ambient and baseline sampling periods. The bimodal distribution corresponds to abrupt temporal shifts in ion current from benzene monomers to protonated water clusters. These shifts correspond to switches in benzene gas cylinders rather than changes in ambient specific humidity, indicating the bimodal distribution is driven by trace water vapor contaminants in the benzene gas cylinders.

a bimodal distribution but no difference from each other (Fig. 8). A similar trend in benzene ion current was observed during standard addition and calibration periods. Benzene ion signal shifted between the two modes several times over the course of the research cruise. Step changes in benzene ion signal corresponded to days when reagent-ion gas tanks were switched. Loss in benzene signal corresponded to nearly equal increases in protonated water cluster peaks (0.7 to $39 \%$ total ion current) and vice versa. These abrupt shifts occur independently of ambient specific humidity or other environmental variables. We attribute these shifts to trace levels of condensation in the benzene standard cylinders or water vapor introduced into the gas delivery lines during cylinder exchange. During the previously described laboratory tests (Sect. 3.1.2), benzene remains $>86 \%$ and protonated water clusters peaked at $8.5 \%$ of total ion current at $100 \%$ relative humidity (Fig. 4a). At typical room temperatures $\left(20-24^{\circ} \mathrm{C}\right), 100 \%$ relative humidity is equivalent to specific humidities between 14.6 and $18.8 \mathrm{~g} \mathrm{~kg}^{-1}$ ). Minute average specific humidity during HiWinGS peaked at $13.2 \mathrm{~g} \mathrm{~kg}^{-1}$, which suggests the large and abrupt increases in protonated water cluster signal (and corresponding decreases in benzene) are driven by water vapor in the reagent-ion generation source rather than ambient humidity. A less probable explanation for the increase in pronated water cluster signals may be attributed to diffusion of water into the permeable gas delivery lines that spanned over $30 \mathrm{~m}$ from the gas cylinder rack on the main deck to the instrument van on the 02 deck. Despite being sheathed, the lines were invariably exposed to sea spray and condensation during high seas. However, this is inconsistent with step-function changes in benzene and water signal over time. Future work will focus on the use of a 
Table 1. Summary of in-field CI-ToFMS performance on the HiWinGS research cruise for select volatile organic compounds. Isoprene and $\alpha$-pinene were calibrated against in-field standards. DMS figures of merit utilized simultaneous independent DMS mixing ratios from the University of Hawaii's APIMS-ILS. Limit of detection (LOD) was calculated for $1 \mathrm{~s}$ averaging times using normalized sensitivities corresponding to $Q=6 \mathrm{~g} \mathrm{~kg}^{-1}$.

\begin{tabular}{lrrr}
\hline VOC species & Sensitivity & Background $(1 \sigma)$ & LOD $(1 \mathrm{~Hz}, 3 \sigma)$ \\
\hline Dimethyl sulfide & 1.0 to $17.9 \mathrm{ncps} \mathrm{ppt}^{-1}$ & $636 \pm 389 \mathrm{cps}$ & $25 \mathrm{ppt}$ \\
Isoprene & 0.7 to $1.1 \mathrm{ncps} \mathrm{ppt}^{-1}$ & $19.5 \pm 66 \mathrm{cps}$ & $15 \mathrm{ppt}$ \\
$\alpha$-Pinene & 0.8 to $6.3 \mathrm{ncps} \mathrm{ppt}^{-1}$ & $192 \pm 191 \mathrm{cps}$ & $14 \mathrm{ppt}$ \\
\hline
\end{tabular}

purified liquid benzene reservoir that would not need to be exchanged during the course of the cruise.

\subsection{In-field calibration and sensitivity}

As expected, absolute sensitivity to VOC standard additions varied as a function of benzene reagent-ion current and ambient water vapor. Calibration factors were calculated from raw count rates observed during each VOC standard addition adjusted for ambient contributions calculated by interpolating values from bracketed sampling periods. DMS calibration factors were derived from concurrent APIMS-ILS determined mixing ratios $\left(\mathrm{cps} \mathrm{ppt}^{-1}\right)$, as discussed in detail below in Sect. 4.5. Mean absolute sensitivities were 19.4, 2.8, and $6.1 \mathrm{cps} \mathrm{ppt}^{-1}$ for DMS, isoprene, and $\alpha$-pinene, respectively.

Instrument baselines are computed from the mean of raw count rates $(1 \mathrm{~Hz})$ measured during valid instrument zero periods throughout the entire campaign as in (Kercher et al., 2009). Variability in baseline counts are largely driven by fluctuations in the benzene reagent-ion current. Detection limits are calculated as in Bertram et al. (2011) as $3 \sigma$ of a Gaussian fit for all baseline count rates over the entire campaign. A conservative estimate of the CI-ToFMS detection limits, for $1 \mathrm{~Hz}$ sample averages, were calculated as 152,13 , and $42 \mathrm{ncps}$ for DMS, isoprene, and $\alpha$-pinene, respectively (Table 1). Using sensitivities reported for $q=6 \mathrm{~g} \mathrm{~kg}^{-1}$, this translates to $1 \mathrm{~Hz}$ detection limits of 25,15 , and $14 \mathrm{ppt}$, respectively.

After normalizing to the benzene ion current, the humidity dependence of in-field VOC normalized calibration factors (Fig. 9) is consistent with laboratory tests (Fig. 4). However, the values of normalized calibration factors were lower by a factor of 4-7 for each VOC molecule, as is highlighted for $\alpha$ pinene in Fig. 10. Possible explanations include VOC standard degradation, inlet performance (less probable for nonpolar terpenes), or most likely shifts in sensitivity from tuning instrument voltages between the two experiments.

In deployments subsequent to the HiWinGS campaign, infield calibrations have been performed utilizing continuous standard additions of isotopically labeled standards (when commercially available) as developed and used on several previous APIMS-ILS air-sea exchange campaigns (Bandy, 2002; Blomquist et al., 2010; Thornton, 2002).

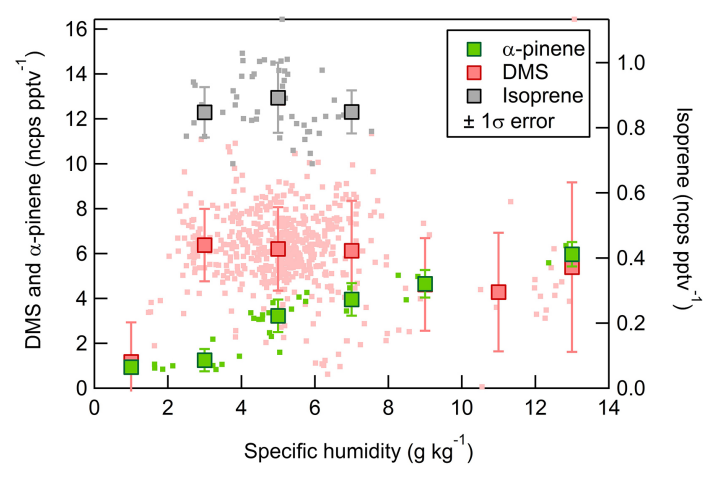

Figure 9. In-field sensitivities during standard additions as a function of ambient specific humidity. Dots represent hourly values while squares represent mean values for a $2 \mathrm{~g} \mathrm{~kg}^{-1}$ specific humidity bin.

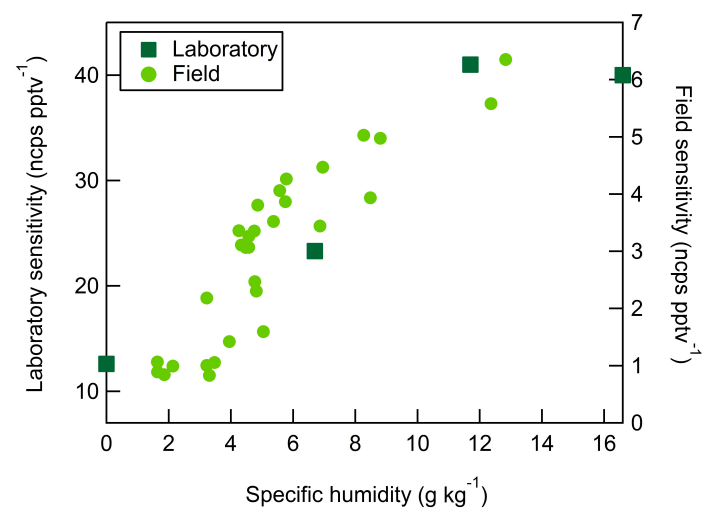

Figure 10. Comparison of $\alpha$-pinene calibration factors observed during laboratory experiments (left axis) and during in-field standard additions (right axis). Similar attenuation with specific humidity was observed.

\subsection{Underway dimethyl sulfide comparison}

Baseline-adjusted, reagent-ion normalized count rates at $62 \mathrm{~m} / Q$ were compared to DMS mixing ratio measurements provided by the University of Hawaii's APIMS-ILS. The instruments were housed in separate instrument vans on the 02 deck of the R/V Knorr. The CI-ToFMS sample manifold was mounted to the foremast as described in Sect. 4.1. The 


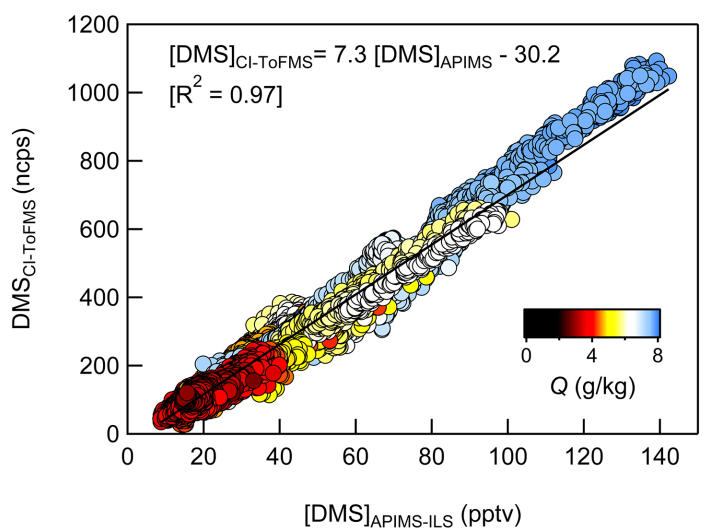

Figure 11. Regression of background corrected, benzene normalized DMS counts with DMS concentrations measured by APIMSILS. Regression analysis indicates a campaign-average CI-ToFMS sensitivity of $6.9 \mathrm{ncps} \mathrm{ppt}^{-1}$.

APIMS-ILS inlet was mounted to the meteorological mast located at the bow of the ship and ca. $16.3 \mathrm{~m}$ a.m.s.l. This is about $17 \mathrm{~m}$ fore and $4 \mathrm{~m}$ below the CI-ToFMS intake position.

Dimethyl sulfide baseline-adjusted, normalized count rates were calculated by subtracting interpolated baseline values from ambient $62 \mathrm{~m} / Q$ counts and scaling to a benzene ion current of $10^{6} \mathrm{cps}$ (Warneke et al., 2001) (Fig. 11). No adjustments based on ambient humidity were made due to the lack of an active standard gas calibration. A regression analysis between the adjusted, normalized CI-ToFMS signal and APIMS-ILS DMS mixing ratio measurements, for $10 \mathrm{~s}$ averages, showed excellent agreement $\left(R^{2}=0.97\right)$ over extreme wind speeds, low ambient loadings, and temperatures below freezing (Figs. 11 and 12). This agreement between two independent DMS measurements lends confidence to the selectivity of both ionization methods and their robustness in the field. It also indicates both sampling locations were functionally equivalent and sampled air masses did not suffer from flow distortion around the superstructure of the ship. As suggested by laboratory tests, the calibration factor is largely constant over the humidity range $\left(1.6-12.8 \mathrm{~g} \mathrm{~kg}^{-1}\right)$ experienced during the cruise (Fig. 12), indicating robustness to ambient humidity fluctuations.

\section{Summary and future directions}

Benzene cluster cations have been revisited as a chemicalionization method for the detection of DMS and select volatile organic compounds. DMS and $\beta$-caryophyllene were identified as novel VOC targets and product ions exhibited limited fragmentation. Sensitivity to DMS, isoprene, and $\alpha$ pinene was determined using CI-ToFMS in laboratory experiments and in-field standard additions with a focus on humidity dependencies for performance in the remote marine boundary layer. Laboratory-based calibration factors ranged
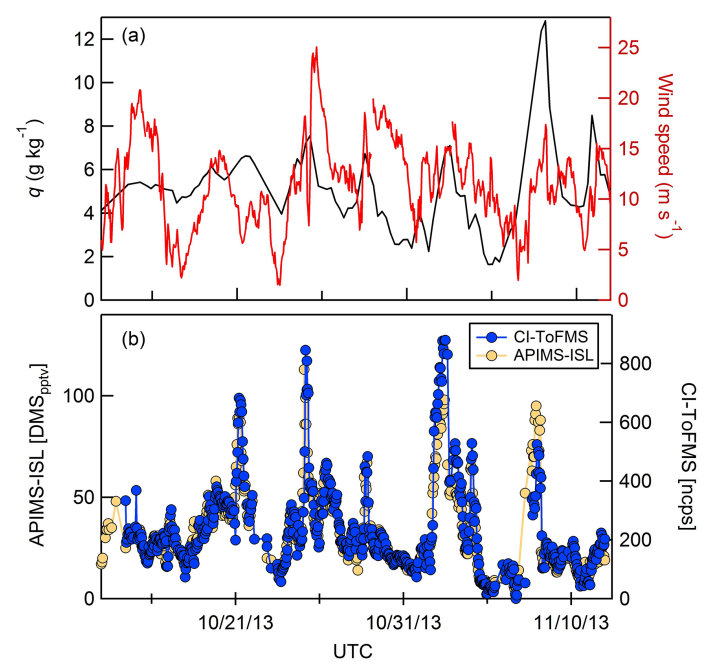

Figure 12. Hourly measurements from the High Wind Gas Exchange Study (HiWinGS) research cruise. Normalized CI-ToFMS count rates compared well $\left(R^{2}=0.80\right.$, hourly averages during entire campaign) with dimethyl sulfide mixing ratios measured by the University of Hawaii's APIMS-ILS. No corrections were made for ambient humidity.

between 2 and $41 \mathrm{ncpspt}^{-1}$, with varying water vapor dependencies. These trends were well conserved between laboratory and field measurements, but normalized sensitivity values were lower in the field by a factor of 4 to 7 . Minor background peaks attributed to alkyl-substituted benzene were traced to contamination in the benzene source. Water vapor in the benzene cylinder standards was also found to degrade benzene reagent-ion signal significantly during field operations. Future directions will explore the use of a liquid benzene reservoir that would not need to be exchanged during the course of the cruise and the potential extension of this ion chemistry beyond the select VOCs studied here.

Acknowledgements. This work was supported by a National Science Foundation (NSF) CAREER Award (grant no. AGS-1151430), the Office of Science (Office of Biological and Environmental Research), US Department of Energy (grant no. DE-SC0006431), and National Aeronautics and Space Administration (NNX12AL94G). The authors thank the entire High Wind Gas Exchange Study science team. A special thanks to the WHOI Marine Operations staff, Captain Kent Sheasley and the capable crew of the R/V Knorr. The authors also thank Emma Mungall and Jonathan Abbatt of the University of Toronto for helpful discussions.

Edited by: J. Abbatt 


\section{References}

Al-Joboury, M. I. and Turner, D. W.: 851. Molecular photoelectron spectroscopy. Part II. A summary of ionization potentials, J. Chem. Soc., 4434-4441, 1964.

Arnold, S. R., Spracklen, D. V., Williams, J., Yassaa, N., Sciare, J., Bonsang, B., Gros, V., Peeken, I., Lewis, A. C., Alvain, S., and Moulin, C.: Evaluation of the global oceanic isoprene source and its impacts on marine organic carbon aerosol, Atmos. Chem. Phys., 9, 1253-1262, doi:10.5194/acp-9-1253-2009, 2009.

Bandy, A. R.: Determination of the vertical flux of dimethyl sulfide by eddy correlation and atmospheric pressure ionization mass spectrometry (APIMS), J. Geophys. Res., 107, 4743, doi:10.1029/2002JD002472, 2002.

Bertram, T. H., Kimmel, J. R., Crisp, T. A., Ryder, O. S., Yatavelli, R. L. N., Thornton, J. A., Cubison, M. J., Gonin, M., and Worsnop, D. R.: A field-deployable, chemical ionization timeof-flight mass spectrometer, Atmos. Meas. Tech., 4, 1471-1479, doi:10.5194/amt-4-1471-2011, 2011.

Blomquist, B. W., Huebert, B. J., Fairall, C. W., and Faloona, I. C.: Determining the sea-air flux of dimethylsulfide by eddy correlation using mass spectrometry, Atmos. Meas. Tech., 3, 1-20, doi:10.5194/amt-3-1-2010, 2010.

Ellis, R. A., Murphy, J. G., Pattey, E., van Haarlem, R., O’Brien, J. M., and Herndon, S. C.: Characterizing a Quantum Cascade Tunable Infrared Laser Differential Absorption Spectrometer (QCTILDAS) for measurements of atmospheric ammonia, Atmos. Meas. Tech., 3, 397-406, doi:10.5194/amt-3-397-2010, 2010.

Goldstein, A. H. and Galbally, I. E.: Known and unexplored organic constituents in the earth's atmosphere, Environ. Sci. Technol., 41, 1514-1521, 2007.

Good, A., Durden, D. A., and Kebarle, P.: Ion-Molecule Reactions in Pure Nitrogen and Nitrogen Containing Traces of Water at Total Pressures 0.5-4 torr. Kinetics of Clustering Reactions Forming $\mathrm{H}^{+}\left(\mathrm{H}_{2} \mathrm{O}\right)_{n}$, J. Chem. Phys., 52, 212-221, 1970.

Grover, J., Walters, E., and Hui, E.: Dissociation energies of the benzene dimer and dimer cation, J. Phys. Chem., 91, 3233-3237, 1987.

Guenther, A. B., Jiang, X., Heald, C. L., Sakulyanontvittaya, T., Duhl, T., Emmons, L. K., and Wang, X.: The Model of Emissions of Gases and Aerosols from Nature version 2.1 (MEGAN2.1): an extended and updated framework for modeling biogenic emissions, Geosci. Model Dev., 5, 1471-1492, doi:10.5194/gmd-51471-2012, 2012.

Helmig, D., Bocquet, F., Pollmann, J., and Revermann, T.: Analytical techniques for sesquiterpene emission rate studies in vegetation enclosure experiments, Atmos. Environ., 38, 557-572, 2004.

Horning, E. C., Horning, M. G., Carroll, D. I., Dzidic, I., and Stillwell, R. N.: New picogram detection system based on a mass spectrometer with an external ionization source at atmospheric pressure, Anal. Chem., 45, 936-943, 1973.

Huey, L. G.: Measurement of trace atmospheric species by chemical ionization mass spectrometry?: speciation of reactive nitrogen and future directions, Mass Spectrom. Rev., 26, 166-184, 2007.

Hunter, E. P. L. and Lias, S. G.: Evaluated Gas Phase Basicities and Proton Affinities of Molecules: An Update, J. Phys. Chem. Ref. Data, 27, 413-656, 1998.

Ibrahim, Y. M., Meot-Ner, M., Alshraeh, E. H., El-Shall, M. S., and Scheiner, S.: Stepwise hydration of ionized aromatics. Energies, structures of the hydrated benzene cation, and the mechanism of deprotonation reactions, J. Am. Chem. Soc., 127, 7053-7064, 2005.

Jaoui, M., Kleindienst, C. T. E., Docherty, B., Lewandoswki, M., and Offenberg, J. H: Secondary organic aerosol formation from the oxidation of a series of sesquiterpenes?: $\alpha$-cedrene,$\beta$ caryophyllene , $\alpha$-humulene and $\alpha$-farnesene with $\mathrm{O}_{3}, \mathrm{OH}$ and $\mathrm{NO}_{3}$ radicals, Environ. Chem., 10, 178-193, 2013.

Kanakidou, M., Seinfeld, J. H., Pandis, S. N., Barnes, I., Dentener, F. J., Facchini, M. C., Van Dingenen, R., Ervens, B., Nenes, A., Nielsen, C. J., Swietlicki, E., Putaud, J. P., Balkanski, Y., Fuzzi, S., Horth, J., Moortgat, G. K., Winterhalter, R., Myhre, C. E. L., Tsigaridis, K., Vignati, E., Stephanou, E. G., and Wilson, J.: Organic aerosol and global climate modelling: a review, Atmos. Chem. Phys., 5, 1053-1123, doi:10.5194/acp-5-1053-2005, 2005.

Kercher, J. P., Riedel, T. P., and Thornton, J. A.: Chlorine activation by $\mathrm{N}_{2} \mathrm{O}_{5}$ : simultaneous, in situ detection of $\mathrm{ClNO}_{2}$ and $\mathrm{N}_{2} \mathrm{O}_{5}$ by chemical ionization mass spectrometry, Atmos. Meas. Tech. 2, 193-204, doi:10.5194/amt-2-193-2009, 2009.

Ketkar, S. N., Dulak, J. G., Dheandhanoo, S., and Fite, W. L.: Benzene charge exchange at atmospheric pressure for low-level detection of pollutants in ambient air, Anal. Chim. Acta, 245, 267 270, 1991.

Kim, S., Karl, T., Helmig, D., Daly, R., Rasmussen, R., and Guenther, A.: Measurement of atmospheric sesquiterpenes by proton transfer reaction-mass spectrometry (PTR-MS), Atmos. Meas. Tech., 2, 99-112, doi:10.5194/amt-2-99-2009, 2009.

Leibrock, E. and Huey, L. G.: Ion chemistry for the detection of isoprene and other volatile organic compounds in ambient air, Geophys. Res. Lett., 27, 1719-1722, 2000.

Lindinger, W., Hansel, A., and Jordan, A.: On-line monitoring of volatile organic compounds at pptv levels by means of protontransfer-reaction mass spectrometry (PTR-MS) medical applications, food control and environmental research, Int. J. Mass Spectrom., 173, 191-241, 1998.

Maleknia, S. D., Bell, T. L., and Adams, M. A.: PTR-MS analysis of reference and plant-emitted volatile organic compounds, Int. J. Mass Spectrom., 262, 203-210, 2007.

McDiarmid, R.: Assignments of Rydberg and valence transitions in the electronic absorption spectrum of dimethyl sulfide, J. Chem. Phys., 61, 274-281, 1974.

Miyazaki, M., Fujii, A., Ebata, T., and Mikami, N.: Infrared Spectroscopy of Size-Selected Benzene-Water Cluster Cations $\left[\mathrm{C}_{6} \mathrm{H}_{6}\right.$ $\left.-\left(\mathrm{H}_{2} \mathrm{O}\right)_{n}\right]^{+}(n=1-23)$ : Hydrogen Bond Network Evolution and Microscopic Hydrophobicity, J. Phys. Chem. A, 108, 1065610660, 2004

Myriokefalitakis, S., Vignati, E., Tsigaridis, K., Papadimas, C., Sciare, J., Mihalopoulos, N., Facchini, M. C., Rinaldi, M., Dentener, F. J., Ceburnis, D., Hatzianastasiou, N., O’Dowd, C. D., van Weele, M., and Kanakidou, M.: Global Modeling of the Oceanic Source of Organic Aerosols, Adv. Meteorol., 2010, 1$16,2010$.

Nemeth, G. I., Selzle, H. L., and Schlag, E. W.: Magnetic ZEKE experiments with mass analysis, Chem. Phys. Lett., 215, 151$155,1993$.

Page, R. H., Larkin, R. J., Shen, Y. R., and Lee, Y. T.: Highresolution photoionization spectrum of water molecules in a supersonic beam, J. Chem. Phys., 88, 2249-2263, 1988. 
Thornton, D. C.: Fast airborne sulfur dioxide measurements by Atmospheric Pressure Ionization Mass Spectrometry (APIMS), J. Geophys. Res., 107, 4632, doi:10.1029/2002JD002289, 2002.

Tonkyn, R. G., Wiedmann, R., Grant, E. R., and White, M. G.: Rotationally resolved photoionization of $\mathrm{H}_{2} \mathrm{O}$, J. Chem. Phys., 95, 7033-7040, 1991.

Warneke, C., van der Veen, C., Luxembourg, S., de Gouw, J., and Kok, A: Measurements of benzene and toluene in ambient air using proton-transfer-reaction mass spectrometry: calibration, humidity dependence, and field intercomparison, Int. J. Mass Spectrom., 207, 167-182, 2001.
Yang, M., Blomquist, B. W., and Nightingale, P. D.: Air-sea exchange of methanol and acetone during HiWinGS: Estimation of air phase, water phase gas transfer velocities, J. Geophys. Res.Oceans, 119, 7308-7323, 2014. 